INVESTIGACIÓN

http://doi.org/10.15198/seeci.2020.51.43-62

Recibido: 26/04/2019 --- Aceptado: 24/06/2019 --- Publicado: 15/03/2020

\title{
MEDIOS DE COMUNICACIÓN Y FLUJOS CULTURALES INTERNACIONALES: LA VIGENCIA ACTUAL DEL INFORME MCBRIDE
}

\section{MEANS OF COMMUNICATION AND INTERNATIONAL CULTURAL FLOWS: THE CURRENT VALIDITY OF THE MCBRIDE REPORT}

\author{
(D) Enrique Vaquerizo Domínguez ${ }^{1}$ : Universidad Complutense de Madrid. \\ España. \\ enrvaque@ucm.com
}

\section{RESUMEN}

Este artículo realiza un análisis de la relación entre los flujos internacionales culturales y comunicativos tomando como referencia las recomendaciones del informe McBride publicado en 1980 como voz de alerta ante la concentración del poder mediático en manos de un reducido número de países, situación que favorecía la uniformización cultural en el planeta. Su objetivo es la actualización de esa fotografía realizada por dicho informe a partir de la disección del origen y control del panorama mediático, así como de sus canales y audiencias principales. A través de un análisis cualitativo de los principales conglomerados mediáticos y canales televisivos internacionales de más difusión, se establece cómo funcionan en la actualidad los mecanismos de influencia cultural en el planeta. Los resultados de la investigación no difieren sustancialmente de los del informe McBride y evidencian cómo el control de los medios de los principales conglomerados de comunicación continúa en manos de un reducido grupo de países occidentales y que sus mensajes continúan contribuyendo a difundir modelos económicos, políticos y culturales hegemónicos. Pese a la prevalencia de esta dinámica el presente artículo también se hace eco de varias respuestas ensayadas a nivel local, desde la creación de medios de comunicación articulados en función de vínculos culturales y dirigidos en muchos casos, a comunidades migrantes en la diáspora, a la aparición de oportunidades innovadoras ligadas al desarrollo del ecosistema digital de comunicación.

PALABRAS CLAVE: sociedad en red - medios de comunicación - flujos informativos - flujos culturales - identidad cultural - comunicación intercultural - informe McBride.

\section{ABSTRACT}

This article analyzes the relationship between international cultural and communicative flows, taking as a reference the recommendations of the McBride

1 Enrique Vaquerizo Domínguez: Doctor en Comunicación Audiovisual por la Universidad Complutense de Madrid y Licenciado en Periodismo e Historia por la Universidad de Sevilla.

enrvaque@ucm.com 
report published in 1980 as a warning to the concentration of media power in the hands of a small number of countries, a situation that favored uniformity cultural on the planet. These pages aim to update that photograph made by said report from the dissection of the origin and control of the media landscape, as well as its main channels and audiences. Through a qualitative research of the main media conglomerates and the most widely spread international television channels in the world, it is established how the mechanisms of cultural influence on the planet work today. The results of the research do not differ substantially from those of the Mc Bride report and show how the control of the media of the main communication conglomerates continues in the hands of a small group of Western countries and that their messages continue to contribute to the dissemination of economic, political models and hegemonic cultural Despite the prevalence of this dynamic, this article also echoes several responses at the local level, from the creation of communication media based on cultural ties and directed in many cases, to migrant communities in the diaspora, to the appearance of innovative opportunities linked to the development of the digital communication ecosystem.

KEY WORDS: networked society - mass media - information flows - cultural flows - cultural identity - intercultural communication - McBride report.

\section{MEDIOS DE COMUNICAÇÃO E FLUXOS CULTURAIS INTERNACIONAIS: A VIGÊNCIA ATUAL DO INFORME MCBRIDE}

\section{RESUME}

Este artigo realiza uma analises da relação entre os fluxos internacionais culturais e comunicativos tomando como referência as recomendações do informe McBride publicado em 1980 como alerta diante da concentração do poder mediático em mãos de um reduzido número de países, situação que favorecia a uniformização cultural no planeta. Seu objetivo é a atualização dessa fotografia realizada por tal informe a partir da dissecção da origem e controle do panorama mediático, assim como de seus canais e audiências principais. Através de uma análise quantitativa dos principais conglomerados mediáticos e canais televisivos internacionais de maior difusão, se estabelece como funcionam na atualidade os mecanismos de influência cultural no planeta. Os resultados da investigação não diferem substancialmente dos informes de McBride e evidenciam como o controle dos meios dos principais conglomerados de comunicação contínua em mãos de um reduzido grupo de países ocidentais e que suas mensagens continuam contribuindo a difundir modelos econômicos, políticos e culturais hegemônicos. Apesar da prevalência desta dinâmica o presente artigo também dá voz a várias respostas ensaiadas a nível local, desde a criação de meios de comunicação articulados em função de vínculos culturais e dirigidos em muitos casos, a comunidades migrantes na diáspora, a aparição de oportunidades inovadoras ligadas ao desenvolvimento do ecossistema digital de comunicação.

PALAVRAS CHAVE: sociedade em rede - meios de comunicação - fluxos informativos - fluxos culturais - identidade cultural - comunicação intercultural informe McBride. 
Vaquerizo Domínguez, E. Medios de comunicación y flujos culturales internacionales: la vigencia actual del informe McBride

\section{Cómo citar el artículo:}

Vaquerizo Domínguez, E. (2020). Medios de comunicación y flujos culturales internacionales: la vigencia actual del informe McBride. [Means of communication and international cultural forms: the current validity of the McBride report]. Revista de Comunicación de la SEECI, 51, 43-62.

doi: http://doi.org/10.15198/seeci.2020.51.43-62

Recuperado de http://www.seeci.net/revista/index.php/seeci/article/view/589

\section{INTRODUCCIÓN}

La existencia del concepto de cultura no solo lleva implícito el de comunicación, sino que como señala Giménez (2009, p. 2) su propia expresión implica ya un proceso de comunicación por sí misma. Si tomamos la definición simbólica de la cultura como "pautas de significados", se observa que el término "significados" refiere automáticamente a la comunicación. Los significados constituyen un producto de la relación entre emisor y receptor y son generados para la interpretación y decodificación de un destinatario o grupo de destinatarios concreto. De esta forma los productos culturales, al igual que los comunicativos, están destinados siempre, y de forma invariable, a un público que es el encargado de reinterpretarlos.

Las interacciones comunicativas tienen lugar siempre dentro de un marco cultural compartido, en mayor o menor medida, por los actores participantes en ese proceso. Este marco cultural proporciona símbolos y códigos comunes, cuadros de referencia, así como presupuestos y protocolos de respeto que facilitan la comunicación.

De esta forma parece razonable afirmar que la comunicación se realiza siempre a partir de las pertenencias socioculturales compartidas entre emisor y receptor. Cuando aparecen disonancias culturales entre ambos actores el proceso fracasa. La comunicación implica una transacción o negociación de identidades. Es necesario que exista una información sobra la identidad del interlocutor para realizar un proceso de adaptación a sus códigos comunicativos al mismo tiempo que se modulan los propios. La comunicación opera sobre la identidad y la cultura no sólo en su dimensión particular, sino también en la colectiva. Para que una identidad colectiva llegue a conformarse ha tenido que existir en primer lugar un desarrollo comunicativo entre sus miembros. Estos han ido recreando, seleccionando y decantando rituales, manifestaciones y productos culturales; así como la construcción de una memoria colectiva para que sea trasmitida de generación en generación.

Pese a que se acepta con naturalidad la proposición de que comunicación y cultura son partes constituyentes de una relación estrecha, durante mucho tiempo, los medios de comunicación y sus contenidos fueron tratados en su origen como ajenos al concepto de la cultura, así como elementos desvirtuadores y "contaminantes" de las identidades culturales. La aparición de los mass media estuvo ligada inicialmente al rechazo que inspiraban entre las élites culturales y los defensores de las tradiciones particularistas ligadas al folclore. Estos presupuestos estaban relacionados principalmente con los estudios de la escuela de Frankfurt, Adorno y Horkheimer (1969), los cuales establecieron una línea diferencial entre la 
cultura tradicional y la industrializada, inserta esta última, en el modo de producción capitalista generada por los medios de comunicación de masas.

Posteriormente, gracias a nuevas perspectivas como los Estudios Culturales, se naturalizó el encaje de los mass media y sus mecanismos de producción dentro de la tradición cultural. Con el final del siglo XX ese debate sería desplazado hacia otro foco: la mundialización cultural. Un proceso generado en un contexto de globalización económica y alentado por el impulso de las tecnologías de comunicación e información (en adelante TIC) experimentado en el siglo XXI.

Hoy el flujo cultural que se produce con la transmisión de creencias, hábitos y valores, se ha acelerado de forma significativa gracias al avance tecnológico de los medios de comunicación y a la consiguiente explosión en una gran variedad de canales y formatos. Por otra parte se han multiplicado las posibilidades de acceso a estos por parte de cada vez más países, que antes estaban relegados a un papel de marginalidad dentro del circuito informativo internacional. Sin embargo existen diferentes visiones acerca de qué mensajes e intenciones subyacen detrás de estos canales y sus contenidos, y de cómo afectan a fenómenos complejos como son las relaciones internacionales culturales, las identidades globales o los fenómenos migratorios.

Estas visiones varían desde aquella alineada con las recomendaciones del informe McBride que considera a los medios tras la llegada de la globalización como mediatizadores de la cultura, responsables de su difusión masiva y vehículos, al cabo, al servicio del poder político y la homogeneización cultural, Castells (1998) Eisteinou (2004), Montáñez (2005), Segovia (2005), Martín Barbero (2008), Thompson (2008), Travesedo (2014), García Canclini (2017). Por el contrario la tendencia representada por Honpenhayn (2004), Lévy (2007), Jenkins (2008) entre otros que confía en el papel de los medios de comunicación, principalmente tras la extensión de sus formatos digitales, como herramientas para preservar y difundir culturas minoritarias gracias a sus estructuras reticulares y posibilidades interactivas.

Esta visión tecnófila promulga la superación de los rígidos esquemas tradicionales de flujos comunicativos y culturales gracias a la aparición de la Sociedad en Red. Por último en necesario señalar la existencia de una línea más escéptica sobre las posibilidades de nuevos medios digitales y flujos culturales. En ella se sitúan las investigaciones de Dijk (2006) Thussu (2007) Sandoval (2007), su escepticismo asociado a la brecha digital de acceso que aún permanece entre sus usuarios.

El presente artículo pretende interrogarse sobre la relación de esos flujos de comunicación sobre las culturas e identidades, que coexisten en el planeta, fundamentalmente a través del análisis de los principales actores que controlan los principales conglomerados mediáticos prestando una atención especial a su procedencia. En el contexto globalizador la mundialización de los procesos culturales choca hoy, en muchos casos, con la reacción de ciertos localismos o regionalismos en una lucha simbólica por la conservación de identidades. Estas tensiones escapan en ocasiones a los rígidos esquemas Norte-Sur para representarse en el escenario de los propios países occidentales, principalmente en sus capitales más cosmopolitas. 
Por un lado al aumento de los flujos migratorios ha aumentado significativamente la movilidad de los ciudadanos y su inserción en sociedades multiculturales, a este hecho se ha unido el desarrollo de las TIC en un proceso que favorece la desterritorialización de las culturas que encuentran nuevos soportes digitales para expresarse. Pese a estos procesos de cambio y a un ecosistema cada vez más atomizado y cambiante, los medios de comunicación, ya sean en sus versiones digitales o analógicas, siguen ejerciendo un rol fundamental como mediadores en los procesos de negociación identitaria realizados por parte de sus audiencias.

\section{OBJETIVOS}

A través de las aportaciones de la bibliografía referida a la temática de medios de comunicación y cultura y del análisis detenido de dos indicadores relevantes como son la naturaleza, procedencia y tamaño de los principales conglomerados mediáticos internacionales así como de los canales de televisión más difundidos del planeta, este artículo persigue un objetivo principal: delimitar la procedencia y destino de los mensajes mediáticos en el panorama internacional, deteniéndose en la carga de contenidos simbólicos y culturales que estos transportan.

A partir de este objetivo principal desarrollaré dos objetivos secundarios:

- Dilucidar si, en función de esos flujos, las recomendaciones del informe McBride aún resultan pertinentes.

- Explorar diversas posibilidades de las que disponen aquellos países que aún ocupan un rol secundario en los circuitos informativos para impactar en sus audiencias.

\section{METODOLOGÍA}

El repaso bibliográfico se ha complementado con el método analítico a la hora de establecer una clasificación sobre los principales conglomerados mediáticos internacionales. Esta clasificación se ha basado en los últimos datos disponibles del estudio Zenith Media, datos actualizados y complementados con el estudio minucioso de los sitios en internet de cada corporación. Su análisis proporciona una fotografía precisa sobre la propiedad y origen de los principales emisores comunicativos. Fotografía que revela a su vez la dirección de los flujos culturales en el planeta. Del mismo modo se ha realizado una investigación cualitativa y analítica sobre algunos de los principales canales globales de televisión por cable. Se han seleccionado estos medios al considerarlos representativos respecto a la emisión de flujos culturales debido tanto al volumen masivo de sus audiencias como a su larga tradición de emisores de flujos comunicativos y culturales con dirección Norte-Sur. Una situación sobre la que ya alertara McBride y que permanece vigente aunque actualizada al contexto digital. Esta investigación se ha centrado en exponer diversos aspectos de esos medios como son procedencia, estructura, temáticas, difusión y alcance de sus audiencias. Para la recopilación de estos datos se han incorporado tanto artículos online como la información proporcionada por los propios canales de televisión. 


\section{DISCUSIÓN}

\subsection{Comunicación, cultura y poder}

La perspectiva de los estudios de comunicación interculturales, a finales del siglo XX, pasa a ser interétnica coincidiendo con el debate sobre el Nuevo Orden de la Comunicación. Durante las últimas décadas, numerosas voces de investigadores provenientes de los países del Sur se han alzado contra la "visión etnocéntrica" occidental la cual no tenía en cuenta las diferencias culturales, sociales y económicas de los países en vías de desarrollo que, siempre desde esta perspectiva, sufrían una "colonización informativa" (Zorogastua, 2015, p. 7).

Según Hall y du Gay (2003), el concepto de identidad no es singular, sino que se construiría en comunidad a través de una multiplicidad de prácticas sociales. Este autor define dos elementos fundamentales en el proceso de formación identitaria: por un lado la influencia del discurso como elemento aglutinador y por otro el concepto de alteridad. Las identidades se construyen a partir de la diferencia en relación, pero también en oposición, a los otros. En esa oposición y reconocimiento de la alteridad, intervienen con un papel muy relevante las relaciones de desigualdad y poder.

Los valores identitarios grupales se articulan en torno a la pertenencia a una comunidad y al discurso que la sustenta. Un relato referido a la fundación o a los orígenes, usos y rituales y a toda una trama de símbolos culturales a los que los medios de comunicación refuerzan, ordenan y dotan de sentido. Los medios, lejos de estar desprovistos de una dimensión cultural, contribuyen al mantenimiento de un determinado orden social y político a través de la emisión de una serie de mensajes más o menos intencionados.

Por otra parte estos canales de comunicación, suelen reflejar el modelo cultural e ideológico dominante de la sociedad a la que pertenecen pese a su aparente "pluralidad" informativa (Hall, 1994). Desde la aparición de los medios de comunicación de masas, y a lo largo de casi toda su historia, la propiedad de estos, y en consecuencia la emisión de mensajes con su carga inherente de símbolos, hábitos y modelos, han estado en manos del espacio cultural y geográfico que se denomina como "civilización occidental".

Desde la aparición de la industria cultural y los medios de comunicación de masas, los flujos tanto comunicativos como culturales, circularon durante décadas, de manera horizontal entre el primer mundo occidental y capitalista hacia el segundo mundo comunista, un eje que se desplazaría con el tiempo hacia una nueva dirección: Norte-Sur. Este último espacio, correspondiente al llamado "tercer mundo", formado por países en vías de desarrollo situados mayoritariamente en África, Asia y Latinoamérica. Las noticias sobre los territorios periféricos estuvieron controladas por las metrópolis durante años y seleccionadas a través del filtro de las corresponsalías y las agencias de noticias, la mayoría en poder de Norteamérica y algunos países europeos.

Esta situación se ha perpetuado hasta las postrimerías del siglo XX, reforzada por el desarrollo cada vez mayor de los mass media, que como todas las instituciones de difusión cultural, están sujetos a toda una serie de determinaciones, la más 
definitoria de ellas, su vinculación con la ideología de la elite dominante. Hall (1972, p. 1) alude a este fenómeno cuando señala que "en Inglaterra, las instituciones de radiodifusión tienen una gran autonomía formal desde el Estado y el gobierno; pero la autoridad última para programar los medios deriva del Estado y, en última instancia, es el Estado de quien son responsabilidad".

Esta tendencia se intensificaría a partir de los años noventa con el boom de la distribución de canales vía satélite globales, principalmente pertenecientes a países europeos, que consiguieron llegar de forma instantánea a hogares de todo el mundo. BBC World, RAI, Internacional, TVE Internacional... todos surgieron como canales alineados con los objetivos geoestratégicos de los países a los que pertenecían con el objetivo de difundir su cultura e incidir en la opinión pública de terceros países en los que emitían Tulloch (2009). Al mismo tiempo, este escenario permitía procesos de impacto e identificación de las audiencias en función de variables de afinidad como la lengua y la cultura por encima de las fronteras geográficas.

Estos patrones, en cuanto a la dirección de los flujos culturales, parecen no haber cambiado hoy sustancialmente. Pese a que es innegable que el mundo globalizado es cada vez más interdependiente y que los avances de las tecnologías de la comunicación juegan un papel fundamental en ese esquema. Como señala Travesedo (2014, p. 545), "la estructura mediática a nivel global ha dado como resultado la aparición de grandes corporaciones influenciadas por políticas nacionales, enfocadas a expandir sus ideas, mensajes, estilos de vida e ideologías".

El peso económico y político se sigue dirimiendo en la capacidad de las diferentes naciones y bloques geopolíticos para transmitir sus ideas, y los nuevos medios de comunicación son una herramienta eficaz para conseguirlo. La estructura del sistema de información a nivel internacional se relaciona de forma indisoluble con los ámbitos de poder e influencia en el ámbito político.

\subsection{El informe McBride, las primeras voces de alarma}

Las primeras voces de alarma respecto a la desigualdad de los flujos informativos se asocian de forma inequívoca al Informe McBride (1980). Este documento supuso un ambicioso proyecto de estudio de los efectos a nivel internacional que provocaban la concentración de los medios de comunicación y el control de los flujos informativos. Según sus tesis, el derecho a la libre circulación de información, convertiría a los medios de comunicación, en instrumentos al servicio de la dominación cultural del conjunto de estados que controlaban la emisión de flujos informativos y los propagaban hacia el resto del planeta.

El informe McBride alertaba sobre cómo las informaciones procedentes de los países desarrollados fluyen hacia los menos desarrollados, sin posibilidad de vuelta. Sus advertencias se resumían en la idea de que la concentración del poder mediático, en manos de un reducido número de países, terminaba ejerciendo a su vez un poder ideológico con influencia en los flujos de ideas y opiniones que acababan por modificar las culturas de los países en desarrollo favoreciendo una 
uniformización cultural en todo el mundo. Ante tal desequilibrio, se proponían recomendaciones a favor de una democratización de la comunicación, en el plano global y nacional, basadas principalmente en una descentralización de los medios de comunicación y en la protección de las culturas minoritarias alentando su propio desarrollo mediático.

Durante estos casi cuarenta años, transcurridos desde el informe McBride, los medios de comunicación de masas han emprendido el camino contrario a sus recomendaciones; una concentración cada vez más evidente, integrada en la lógica de mercado capitalista donde el tamaño favorece la competitividad. Segovia (2005), sostiene que la búsqueda del crecimiento constante habría llevado a los medios de comunicación a una serie de estrategias como monopolización de mercados, búsqueda de nuevos nichos, potenciación de la imagen de marca, diversificación de productos y control de cada vez un mayor número de pasos en la cadena de producción.

Ante la cada vez mayor integración de los medios de comunicación en la lógica capitalista chasta qué punto los canales de comunicación transmiten hoy lenguas, valores y modos de vida de culturas minoritarias? 0 , por el contrario ¿siguen ejerciendo como instrumentos al servicio de la homogeneización cultural procedente de los países occidentales como señalara McBride, hace ya más de 40 años? La respuesta, sobre todo tras la generalización del uso de internet y los procesos de convergencia mediáticos en la red presenta cada vez más matices.

Hoy en la relación entre cultura y comunicación vivimos un tiempo marcado por la hibridación entre lo local y lo global, pero también una hibridación tecnológica y mediática, en la que los contenidos informativos culturales se desarrollan y consumen envueltos en dinámicas transmedia y a partir de dispositivos cada vez más sofisticados. Existe una corriente optimista propugnada por autores como Honpenhayn (2004), Lévy (2007), Jenkins (2008) que destaca los efectos que la globalización y la llegada de las TIC ha generado en la capacidad de los receptores para influir en el proceso comunicativo, el entorno digital habría favorecido la difuminación de las fronteras entre emisores y receptores provocando un descentramiento respecto a las jerarquías, los espacios y los soportes que establecía la comunicación de masas y que determinaba la relación entre emisor y receptor.

\subsection{Los flujos culturales y mediáticos en la actualidad}

A pesar de que la comunidad internacional está cada vez más interconectada, gracias en parte a la proliferación y diversidad de acceso a nuevos medios de comunicación, en plena Sociedad de la Información la emisión de flujos comunicativos y por ende culturales sigue ligada a la capacidad tecnológica y a los intereses geopolíticos de los países. Siguiendo la teoría de los flujos de Castells (1999, p. 114), "la generación de riqueza, el ejercicio del poder y la creación de códigos culturales han pasado a depender de la capacidad tecnológica de las sociedades y las personas, siendo la tecnología de la información el núcleo de esta capacidad". De esta forma ¿Seguimos en un escenario en el que los principales 
Vaquerizo Domínguez, E. Medios de comunicación y flujos culturales internacionales: la vigencia actual del informe McBride

conglomerados mediáticos y flujos comunicativos pertenecen a los países del Norte? ¿Tienen vigencia hoy las recomendaciones del informe McBride para alcanzar un modelo más igualitario desde el punto de vista informativo y cultural?

Tabla 1. 25 principales conglomerados mediáticos del mundo.

\begin{tabular}{|c|c|c|}
\hline Corporación & Nacionalidad & Medios principales \\
\hline Alphabet & Estadounidense & $\begin{array}{l}\text { Empresa subsidiaria de Google, trabaja con } \\
\text { aplicaciones, servicios relacionados con Google } \\
\text { (YouTube, Adwords, Drive, Android.... }\end{array}$ \\
\hline Facebook & Estadounidense & $\begin{array}{l}\text { Redes Sociales y mensajería instantánea: } \\
\text { (WhatsApp, Instagram). }\end{array}$ \\
\hline Comscat & Estadounidense & $\begin{array}{l}\text { Servicios televisivos por cable, Internet y } \\
\text { telefonía. }\end{array}$ \\
\hline Baidu & China & $\begin{array}{l}\text { Motor de búsqueda y almacenamiento de datos } \\
\text { chino con más de } 500 \text { millones de usuarios. }\end{array}$ \\
\hline $\begin{array}{l}\text { The Walt Disney } \\
\text { Company }\end{array}$ & Estadounidense & $\begin{array}{l}\text { Compañía de medios y entretenimiento con } \\
\text { canales de televisión y estudios de cine, Walt } \\
\text { Disney Studios, Walt Disney Pictures, Walt } \\
\text { Disney Animation Studios, Pixar Animation } \\
\text { Studios, Disneynature, 20th Century Fox, Blue } \\
\text { Sky Studios, Disney Channel, Fox Channel. } \\
\end{array}$ \\
\hline CBS Corporation & Estadounidense & $\begin{array}{l}\text { Canales y productoras de televisión como The } \\
\text { CW television Network Showtime Simon \& } \\
\text { Schuster Columbia Records. }\end{array}$ \\
\hline Microsoft & Estadounidense & $\begin{array}{l}\text { Soporte de software para computadores } \\
\text { personales, servidores, dispositivos electrónicos } \\
\text { y servicios (Internet Explorer, Windows, Skype, } \\
\text { Xbox Game Studies, redes sociales como } \\
\text { LinkedIn). }\end{array}$ \\
\hline Bertelsmann & Alemán & $\begin{array}{l}\text { Radiotelevisón y empresa editorial: Canales y } \\
\text { productoras de televisión alemanas RTL y VOX, } \\
\text { Penguin Random House la mayor editorial del } \\
\text { mundo, Gruner + Jahr (editorial de revistas } \\
\text { como GEO o Sterm). }\end{array}$ \\
\hline Viacom & Estadounidense & $\begin{array}{l}\text { Productoras (Paramount Pictures), televisión } \\
\text { por cable y satélite (MTV Networks, } \\
\text { BETy Nickelodeon) e Internet (Neopets). }\end{array}$ \\
\hline WarnerMedia & Estadounidense & $\begin{array}{l}\text { Plataformas de distribución de contenidos, } \\
\text { como HBO GO y Max GO, canales } \\
\text { internacionales de noticias, entretenimiento, } \\
\text { animación y deportes como CNN, HLN, TNT, } \\
\text { TBS, Cartoon Network, productoras de cine } \\
\text { como Warner Bros, revistas como Time o } \\
\text { Sports Ilustrated. }\end{array}$ \\
\hline Verizon & Estadounidense & $\begin{array}{l}\text { Telefonía móvil con filiales como AOL y } \\
\text { buscadores tras la compra de Yahoo!. }\end{array}$ \\
\hline Tencent & China & $\begin{array}{l}\text { Productos de servicios y publicidad en internet } \\
\text { redes sociales, mensajería instantánea, } \\
\text { comercio electrónico con marcas como QQ, } \\
\text { SOSO o WeChat. }\end{array}$ \\
\hline Hearst & Estadounidense & $\begin{array}{l}\text { Periódicos, más de } 50 \text { cabeceras diarias y } \\
\text { semanales entre las que destacan algunas } \\
\text { como San Francisco Chronicle o medios étnicos }\end{array}$ \\
\hline
\end{tabular}


Vaquerizo Domínguez, E. Medios de comunicación y flujos culturales internacionales: la vigencia actual del informe McBride

\begin{tabular}{|l|l|l|}
\hline & & $\begin{array}{l}\text { como La Voz o Nuestra Gente, participación } \\
\text { con un 20\% en el canal de televisión ESPN. }\end{array}$ \\
\hline Advance Publications & Estadounidense & Periódicos, webs de información y televisión. \\
\hline JCDecaux & Francia & Publicidad exterior. \\
\hline News Corporation & Estadounidense & $\begin{array}{l}\text { Medios de Comunicación, internet y } \\
\text { entretenimiento, periódicos como The Sun o } \\
\text { New York Post, noticias empresariales Dow } \\
\text { Jones Media Group, Fox Broadcasting } \\
\text { Company, Sky, ITV o Star TV. }\end{array}$ \\
\hline Grupo Globo & Brasil & $\begin{array}{l}\text { Canales de televisión por cable o satélite, } \\
\text { periódicos y empresas editoriales O Globo, } \\
\text { Globo Sat, Globo News. }\end{array}$ \\
\hline CCTV & China & $\begin{array}{l}\text { Televisión Central China grupo público de } \\
\text { comunicación con canales de televisión, entre } \\
\text { ellos canales internacionales, con CCTV-4 como } \\
\text { el destinado para la diáspora china. }\end{array}$ \\
\hline Mediaset & $\begin{array}{l}\text { Televisiones en Italia y España con canales } \\
\text { como Cuatro, Telecinco, Canale 5 o Italia 1. }\end{array}$ \\
\hline $\begin{array}{l}\text { Discovery } \\
\text { Communications }\end{array}$ & Estadounidense & $\begin{array}{l}\text { Canales de televisión temáticos: Discovery } \\
\text { Channel, Animal Planet, Travel Channel. }\end{array}$ \\
\hline TEGNA & Estadounidense & $\begin{array}{l}\text { Servicios de transmisión, medios digitales y } \\
\text { marketing. }\end{array}$ \\
\hline ProSiebenSat.1 Group & Alemán & Televisión, radio e internet. \\
\hline $\begin{array}{l}\text { Sinclair Broadcasting } \\
\text { Group }\end{array}$ & Estadounidense & Televisión, radio y medios digitales. \\
\hline Axel Springer & Alemán & Periódicos revistas y medios digitales. \\
\hline $\begin{array}{l}\text { Scripps Networks } \\
\text { Interactive }\end{array}$ & Estadounidense & $\begin{array}{l}\text { Canales vía satélite e internet: Canal Cocina, } \\
\text { Travel Channel o Asian food Channel entre sus } \\
\text { canales más conocidos. }\end{array}$ \\
\hline
\end{tabular}

Fuente: elaboración personal a partir del informe de datos de Zenith Media en 2017.

Pese a estos tímidos cambios en el panorama audiovisual, experimentados durante los últimos años, si atendemos a los últimos datos disponibles, un estudio de Zenith Optimedia (2017), 18 de los 25 principales conglomerados de comunicación a nivel mundial están radicados en los Estados Unidos y el mayor porcentaje de su capital es estadounidense. Google, Facebook, Walt Disney Company, CBS Corporation, Comcast, Alphabet pertenecerían a lo que podríamos denominar como cultura anglosajona. Junto a ellas, entre las 25 corporaciones principales, figurarían tres procedentes de China, tres de Alemania, una francesa y una italiana. Desde América Latina, la única que figura en los puestos principales sería la brasileña Globo, S.A., ubicada en el puesto número 17.

La mayoría de esos conglomerados ha aceptado con naturalidad la llegada y generalización del uso de la red, la inserción en el ecosistema digital y la distribución de contenidos transmedia. La "convergencia" tecnológica preconizada por Honpenhayn (2004) ha provocado megafusiones empresariales que han integrado las nuevas oportunidades. De esta forma si la llegada de la Comunicación de Masas difuminó los límites entre la alta y baja cultura, hoy los medios audiovisuales convencionales y los nuevos medios interactivos se encuentran inmersos en un nuevo proceso de concentración. 
Así en esta clasificación coexisten corporaciones especializadas en medios de comunicación audiovisuales, con productoras, prensa escrita y editoriales con aquellas que trabajan con navegadores web, aplicaciones, redes sociales o servicios de mensajería instantánea. Sin embargo el paso de la Comunicación de Masas a la Sociedad en Red no parece haber cambiado el origen y la propiedad de canales, emisores y mensajes, estos parecen concentrados salvo excepciones en los mismos países que hace cuarenta años cuando el informe McBride manifestó las primeras señales de alarma.

Tabla 2. Canales de televisión con más difusión del planeta.

\begin{tabular}{|c|c|c|}
\hline Canal & Nacionalidad & Temática \\
\hline BBC News & Reino Unido & Noticias \\
\hline ESPN & $\begin{array}{l}\text { Estadounidense (Propiedad de } \\
\text { Walt Disney Company) A través } \\
\text { de ESPN Latinoamérica realiza } \\
\text { retransmisiones de eventos } \\
\text { deportivos en este continente. }\end{array}$ & Retransmisión de eventos deportivos. \\
\hline $\mathrm{HBO}$ & Estadounidense & Series de entretenimiento. \\
\hline NBC News & Estadounidense & Noticias. \\
\hline $\begin{array}{l}\text { CW Television } \\
\text { Network }\end{array}$ & Estadounidense & $\begin{array}{l}\text { Series de televisión, realities y } \\
\text { programación infantil. }\end{array}$ \\
\hline Channel V & $\begin{array}{l}\text { Sede en Hong Kong pero } \\
\text { propiedad estadounidense } \\
\text { propiedad de The Walt Disney } \\
\text { Company Asia Pacific } \\
\text { subdivisión de The Walt Disney } \\
\text { Company }\end{array}$ & $\begin{array}{l}\text { Televisión de pago con contenidos } \\
\text { musicales y series dirigido al público de Asia } \\
\text { Pacífico con subdivisiones para India, } \\
\text { Tailandia, China, Australia... }\end{array}$ \\
\hline Star World & $\begin{array}{l}\text { Sede en Hong Kong pero capital } \\
\text { estadounidense, propiedad de } \\
\text { Walt Disney Company. }\end{array}$ & $\begin{array}{l}\text { Programas de entretenimiento emitidos en } \\
\text { inglés y dirigido a la población del sudeste } \\
\text { asiático de habla inglesa. }\end{array}$ \\
\hline BBC Food & Reino Unido & $\begin{array}{l}\text { Canal de cocina propiedad de BBC } \\
\text { Corporation. }\end{array}$ \\
\hline FOX & Estadounidense & $\begin{array}{l}\text { Canal de series, películas y documentales } \\
\text { propiedad de Walt Disney Corporation. }\end{array}$ \\
\hline MTV & $\begin{array}{l}\text { Estadounidense, propiedad de } \\
\text { VIACOM con más de } 20 \\
\text { divisiones internacionales }\end{array}$ & Cadena de videos musicales. \\
\hline Disney Channel & $\begin{array}{l}\text { Estadounidense, subdivisiones } \\
\text { en Latinoamérica, Sudáfrica, } \\
\text { Asia, Europa, India, Australia y } \\
\text { Rusia. }\end{array}$ & $\begin{array}{l}\text { Dibujos animados y series destinadas a } \\
\text { público infantil y adolescente. }\end{array}$ \\
\hline $\begin{array}{l}\text { Discovery } \\
\text { Channel }\end{array}$ & $\begin{array}{l}\text { Estadounidense, propiedad de } \\
\text { Discovery INC y con } \\
\text { subdivisiones en Europa, Asia, } \\
\text { Australia y Canadá. } \\
\end{array}$ & Documentales de Naturaleza. \\
\hline Canal Historia & $\begin{array}{l}\text { Estadounidense, propiedad de } \\
\text { Hearts Corporation con } \\
\text { subdivisiones en Europa, } \\
\text { Latinoamérica, Medio Oriente, } \\
\text { Canadá, la India y Australia. }\end{array}$ & Documentales históricos. \\
\hline
\end{tabular}

Fuente: elaboración propia a partir de informaciones de Marketing91 y Trendrr.net 
Al analizar al difusor por excelencia de flujos culturales como es la televisión vía satélite y tomando como referencia a algunos los canales más vistos en los hogares de todo el mundo: BBC, HBO, MTV, Disney Channel o ESPN, se observa cómo la práctica totalidad pertenecen también a conglomerados británicos 0 estadounidenses. La mayoría de ellos disponen de subdivisiones para Latinoamérica, Asia e incluso África en los que los contenidos se adaptan al lenguaje y, en ocasiones, supuestos intereses de sus audiencias. Fuera del ámbito occidental solo destacan Star TV o Channel $V$ canales radicados en Hong Kong y dirigidos exclusivamente al público del sudeste asiático pero que también pertenecen a conglomerados occidentales.

De esta forma, algunos los canales de televisión referencia relacionados con algunos de los aspectos más relevantes para la conformación de una cultura como son la información, la lengua y la Historia e incluso la gastronomía, la educación infantil, la música y el deporte, están controlados hoy por capital anglosajón que decidiría las directrices y la programación de contenidos que se consumen en los hogares de todo el mundo.

En el caso estadounidense, siguiendo a Segovia (2004), sus conglomerados mediáticos, en la mayoría de los casos están participados por otros sectores como la banca o el petróleo y se ven apoyado por políticas públicas que apoyan su desarrollo. Esas políticas han privilegiado tradicionalmente la información como un actor de enorme influencia en la geopolítica internacional, en el que la dominación cultural se une a otras como la militar o la económica.

\subsection{La sociedad en red: ¿nuevo paradigma de flujos mediáticos y culturales?}

Aunque algunas de las conclusiones del informe McBride han quedado obsoletas y no bastan para explicar en su totalidad la realidad informativa de siglo XXI, es cierto que los flujos informativos siguen proviniendo principalmente de los países desarrollados. Sin embargo también se ha ampliado el acceso a las fuentes de información con el desarrollo de la red y la generación de nuevos canales propios, implementados por países que antes pertenecían a circuitos informativos secundarios. A través de la revolución tecnológica de las últimas décadas y la proliferación de medios de comunicación, una gran mayoría de comunidades que antes vivían aisladas, se encuentran hoy interconectadas. En este proceso, junto a internet, la televisión no ha perdido relevancia, como modificadora de aspiraciones sociales y modelos de comportamiento, económicos y culturales de sociedades dónde estos habían permanecido inalterados durante siglos.

Desde este punto de vista, la dominación de la identidad cultural permanece ligada a los intereses económicos y políticos y se reproduce, como precisa Castells (1996, p. 211), a través de los flujos globales y abstractos de riqueza, poder e información, que construyen la virtualidad real mediante redes de medios de comunicación. La apertura de los polos de influencia política y económica hacia naciones emergentes lleva aparejada la aparición de medios globales con sedes en 
países que tradicionalmente habían ocupado el papel de meros receptores, cambios que se han traducido sobre todo en el panorama audiovisual. Durante las dos últimas décadas, se han producido los primeros flujos informativos Sur-Sur y una significativa difuminación de los conceptos de centro y periferia.

Algunos países considerados tradicionalmente periféricos en los circuitos de información hace años que empezaron a consolidarse como exportadores de productos culturales, principalmente de carácter audiovisual, dirigidos a nichos de población con los que comparten afinidad identitaria. Destacan los casos de Al Jazeera, Telesur, CCTV o Univisión, canales que se dirigen a audiencias amplias con las que comparten lengua, procedencia o identidad cultural.

Entre esos nichos se encuentran las minorías de migrantes residentes en sociedades cada vez más multiculturales. Estos canales de televisión funcionan en muchos casos como soporte para la conexión diaspórica de una comunidad dispersa por el mundo o simplemente desplazada de sus lugares de origen a otros emplazamientos más o menos cercanos. En algunos casos, este uso viene motivado por el interés de diferentes gobiernos en mantener viva la relación entre los emigrantes y su país, entre otras, por razones de tipo económico como es el caso del canal público chino CCTV y de la diáspora china en el mundo (Amezaga, 2004, p. 8).

En la actualidad los grupos diaspóricos tienen, por primera vez, la posibilidad de acceder a medios de comunicación procedentes de sus comunidades de origen que permiten consumir contenidos en su propia lengua, asistir a una ceremonia religiosa o seguir la misma serie que visionaba en su país de origen sin salir de casa. Para ellos, se han creado también nuevos canales de televisión, retransmitiendo desde la propia sociedad de acogida. Así se han extendido casos como los de Hispan TV o Córdoba Internacional, canales de televisión musulmanes emitidos para la comunidad musulmana en países de habla hispana, que intentan también difundir propaganda entre sus audiencias sobre el régimen de Irán y la monarquía saudí respectivamente.

Con la apertura del panorama televisivo, a través de la inclusión ocasional de canales audiovisuales dedicados a minorías dentro de los grandes conglomerados internacionales, se producen también ciertos contraflujos audiovisuales y culturales desde el Sur hacia el Norte, aunque estos flujos aún sean minoritarios. Las telenovelas, como señala Thussu (2007, p. 48), son un ejemplo en ese sentido; producidas en México, Colombia, Brasil y Venezuela, son transmitidas a través de sus respectivas corporaciones por canales dedicados a minorías étnicas y culturales, pero que se emiten en abierto en todo el territorio estadounidense, como es el caso de Latino TV.

A pesar de ese cambio de tendencia en los flujos audiovisuales, autores críticos como Gómez (2016) señalan que estos contenidos ayudan a reflejar estereotipos culturales y no se emiten en los principales canales nacionales en Estados Unidos, sino en mercados culturales secundarios basados en un componente diaspórico y étnico y destinados en muchos casos a la penetración comercial en el nicho al que representan. Del mismo modo, esta diversificación de los grandes conglomerados mediáticos hacia divisiones 
regionales, o la adaptación de sus contenidos para llegar a minorías étnicas, obedecen más a una exploración de nuevas audiencias estandarizadas que a una dirección real hacia la democratización de los flujos culturales.

La llegada de las TIC sí parece una posibilidad de alterar el flujo cultural unidireccional de la comunicación de masas sobre el que alertaba el informe McBride. Estas herramientas como sostiene García Canclini (2017, p. 19) se han convertido en mediadores de la comunicación y la cultura y una oportunidad para todas las comunidades que pretendan buscar su reconocimiento sociocultural. Sin embargo a pesar de los cambios que comienzan a esbozarse, aún persisten dudas sobre si el ciberespacio seguiría contribuyendo a consolidar la hegemonía cultural de las naciones que controlan los conglomerados mediáticos tradicionales, o si también permite la presencia, de otras culturas no occidentales.

La llegada del ecosistema digital trajo aparejado el término cibercultura Lévy (2007, p. 12), concebido bajo la hibridación tecnológica, mediática y cultural. Un espacio digital y abierto para la producción y consumo a través de redes colaborativas y horizontales, formadas por usuarios que lograban romper las rígidas fronteras entre emisor y receptor marcada por la tradicional comunicación de masas. En la actualidad, los individuos pueden asociarse en comunidades virtuales como las redes sociales en función de sus intereses y afinidades culturales y contribuir de forma democrática con contenidos rompiendo, en apariencia, el monopolio de los medios de comunicación sobre su producción. Sin embargo, los medios de comunicación siguen teniendo un gran peso en la generación de esos contenidos que suponen un combustible necesario para la interacción de estos espacios.

En conjunto, a pesar de las nuevas tendencias comunicativas esbozadas en este capítulo y las posibilidades que ofrecen las TIC subrayadas por tecnófilos como Honpenhayn (2004) o Lévy (2007), la unidireccionalidad y concentración de los medios de comunicación a nivel mundial, con la consiguiente uniformización cultural en la emisión de mensajes, continúan vigentes y vinculados a los centros productores de riqueza (Travesedo, 2014, p. 9). A pesar de los considerables efectos de la revolución digital, continúan existiendo grandes segmentos de población que ocupan el papel de meros receptores de flujos culturales o que en ocasiones, ni tan siquiera pueden acceder a ellos.

En el acceso a la información a través de las TIC, pese a la tendencia a elevar a un nivel exclusivamente "metafísico" las interacciones virtuales y a minimizar la importancia de los dispositivos, coexisten permanentemente los elementos físicos y virtuales; como señala Sandoval (2007, p. 14), no todo lo que ocurre en el ciberespacio está apartado de la realidad cotidiana.

Para producir interacciones en una comunidad virtual es necesario un sustrato tecnológico como una pantalla, un teléfono, una conexión... El acceso a ellos viene determinado por las condiciones económicas de los individuos, posición social, situación laboral, grupo o grupos de pertenencia, idioma, cultura y religiosa. Estas variables terminan por condicionar, de forma fundamental, la sociabilidad virtual de los individuos. 
El carácter democrático y horizontal de las TIC no reproduce, de forma automática, una condición de igualdad de los individuos en la Sociedad de la Información.

Si la desigualdad en la dirección de los flujos de comunicación ha persistido hasta nuestros días también existen desigualdades de acceso a las TIC que, pese a un claro retroceso en las últimas décadas, aún presentan matices que influyen en la posición que una comunidad ocupa en la Sociedad de la Información. El concepto de brecha digital, que podríamos definir desde su perspectiva clásica como "la distancia entre aquellas personas que tienen y no tienen acceso a internet" (Dijk, 2006, p. 221), sirve para ilustrar este fenómeno. La brecha digital representa el impacto social disímil que provocan las TIC, así como las distancias que se establecen en cuanto a las oportunidades de desarrollo de las poblaciones que las utilizan.

La utilización de la tecnología puede marcar una frontera entre "ricos" y "pobres", con el acceso a la información como capital diferenciador. En este contexto, sólo los privilegiados son capaces de cosechar los beneficios sociales y económicos del acceso a la infraestructura mundial de la información y las comunicaciones. Esta nueva forma de exclusión se reproduce a través de dos vertientes: brecha digital internacional (abismo que separa a las regiones y a los países) y brecha digital doméstica (divide a los grupos de ciudadanos de una sociedad) (CEPAL, 2003). La brecha digital separaría a los que están conectados a la sociedad digital a través de las TIC de los que no tienen acceso a los beneficios de las nuevas tecnologías y se produce, como señala Tello $(2007$, p. 3), a través de las fronteras internacionales, pero también dentro de las comunidades locales divididas por las barreras económicas y de acceso al conocimiento.

A lo largo de los últimos años, las tasas de penetración de internet han alcanzado índices muy elevados, provocando un retroceso significativo de la brecha digital, pese a que aún persistan algunas diferencias. Según datos de la consultora Nielsen (2017), los índices de penetración de internet para Norteamérica y Europa se cifrarían en un 88 y un $77 \%$, respectivamente, frente al $59 \%$ de Latinoamérica y el $28 \%$ de África. Esta última ha crecido a un ritmo superior al $1.000 \%$ durante los últimos años, por lo que es presumible que esa diferencia a pesar de ser aún significativa quede subsanada en poco tiempo.

Ahora, los estudios más recientes sobre la brecha digital no se limitan a evaluar el acceso a los servicios que ofrece internet, sino que también se tiene en cuenta la calidad de dicho acceso y la disponibilidad de conexiones de banda ancha que permitan acceder a contenidos multimedia en tiempos y costos adecuados al contexto de los usuarios. Dijk (2013), tras estudiar el comportamiento respecto al uso de las tecnologías digitales en la red de cientos de holandeses, concluye que en la capacidad para desenvolverse alrededor de las TIC influyen variables como la edad, la etnia o la educación, y que extraer el máximo rendimiento de estas, repercute favorablemente en el establecimiento de unas redes extensas y ricas en el ámbito offline.

Desde esta óptica, la arquitectura de la brecha digital se establecería a través de dos direcciones: una horizontal en la que la pobreza generaría exclusión digital y otra 
Vaquerizo Domínguez, E. Medios de comunicación y flujos culturales internacionales: la vigencia actual del informe McBride

vertical basada en las políticas que, a través del desarrollo de las infraestructuras, garantizarían, o no, el acceso igualitario a todos sus ciudadanos.

\section{CONCLUSIONES}

Tras el análisis sobre el origen de los principales conglomerados comunicativos del panorama internacional resulta evidente que la mayoría de ellos pertenecen a corporaciones con capital procedente de países occidentales, principalmente anglosajones. La investigación sobre los principales canales vía satélite en el planeta ofrece resultados similares, la mayoría pertenecen a conglomerados británicos y estadounidenses. Aunque algunos de ellos poseen subdivisiones continentales y adaptan contenidos y lenguajes a sus audiencias, su programación suele decidirse en las sedes centrales, propagando flujos culturales e informativos hegemónicos en relación al capital del que proceden. Muchos de estos espacios constituyen canales temáticos centrados en aspectos esenciales para la conformación de una cultura como serían la lengua, la Historia la gastronomía, la educación infantil, la música y el deporte. Sus programas difunden una correspondiente carga de contenidos simbólicos occidentales que son consumidos por audiencias de todo el mundo, en muchos casos pertenecientes a países que siguen ocupando un lugar marginal dentro del juego de la distribución de flujos informativos y culturales.

En relación al segundo objetivo propuesto en esta investigación, comprobar la vigencia de algunas de las recomendaciones del informe MCBride, es necesario señalar que este alertaba sobre el hecho de que la industria de la comunicación estuviese dominada por un número relativamente pequeño de empresas que concentraban casi todo el proceso de producción y la distribución, de vocación transnacional y situadas en los principales países desarrollados. Sus recomendaciones principales incidían en la necesidad de liquidar las barreras internas y externas, que impedían la libre circulación de información, así como garantizar la libertad de prensa, la pluralidad de las fuentes y de canales de la información, algo que la propia eclosión del sistema digital de información y el desarrollo de las TIC, multiplicando la oferta informativa parecen haber limitado al menos en apariencia.

La llegada de internet y el desarrollo económico de algunos de los países tradicionalmente situados en la periferia informativa, también parece haber ayudado a avanzar en otras de las principales líneas de recomendaciones del informe: respetar la identidad cultural y el derecho de cada país de informar a los ciudadanos del mundo sobre sus aspiraciones, valores sociales y culturales, o respetar el derecho de todos los pueblos del mundo a participar en los flujos de información internacionales y de los ciudadanos a acceder a las fuentes de información y de participar activamente en el proceso de comunicación. En la actualidad en el ámbito audiovisual internacional al hablar de flujos informativos y su influencia cultural coexisten diversas situaciones: desde el predominio de los países del Norte como emisores de mensajes persuasivos que continúan propagando, como alertara el informe McBride desde hace décadas, la cultura hegemónica occidental, al desarrollo de nuevas tendencias audiovisuales enfocadas en comunidades culturales muy específicas, que por primera vez tienen la oportunidad de consumir contenidos 
especialmente dirigidos hacia ellas. En ambas situaciones subyace una intencionalidad en la emisión de los mensajes con una clara voluntad geoestratégica.

Pese a los tímidos avances los resultados de esta investigación evidencian que no se han producido avances sustanciales en una de las recomendaciones principales, disminuir el alcance de los monopolios informativos y sus efectos. En el siglo XXI el control y la emisión de la mayoría de mensajes informativos con su correspondiente carga simbólica y de flujos culturales continúan en manos de unos pocos países, favoreciendo una uniformización cultural en todo el mundo como alertaba el informe. En estos cuarenta años, los medios de comunicación de masas han emprendido un proceso de concentración cada vez más evidente. Pese a la diversificación de formatos y contenidos y al aparente empoderamiento de los receptores gracias al paso de una Comunicación de Masas a una Sociedad en Red, los canales y flujos de información continúan en manos en su mayoría de los países occidentales y por tanto las denuncias y recomendaciones que hiciera el informe continúan vigentes. La aparición de las TIC no han provocado la desaparición de los medios tradicionales. Por el contrario en muchos casos los han integrado, diversificando sus usos y modos de consumo, del mismo modo algunas de los espacios más importantes del entorno digital como Facebook o Google son propiedad también de capital occidental que las han integrado en un fenómeno de concentración mediático cada vez más horizontal y diverso.

Entre los objetivos de esta investigación figuraba explorar las diversas posibilidades de las que disponen aquellos países que aún ocupan un rol secundario en los circuitos informativos para llegar a sus audiencias. Si bien el método de investigación escogido no ha permitido profundizar en aspectos como nuevos espacios digitales y tendencias de consumo exclusivos de estos países, dejando espacio para futuras líneas de investigación, la observación de nuevos fenómenos y formas de comunicación digital si permite concluir algunos países tradicionalmente relegados a papeles marginales dentro de los circuitos informativos lanzan canales audiovisuales y comunidades digitales con vocación de influencia geoestratégica y de articulación diaspórica respecto a audiencias de afinidad cultural.

Del mismo modo pese a los condicionantes señalados anteriormente, se puede resaltar que las TIC también han aportado herramientas para aquellas comunidades que tradicionalmente habían permanecido relegadas en los circuitos clásicos tanto culturales como informativos favoreciendo una cierta diversificación. Sin embargo en la práctica, gran parte de la población de esos países continúa teniendo dificultades para aprovechar estas oportunidades, unas dificultades asociadas a la brecha digital tanto de acceso como de uso. De esta forma el panorama sobre el control y emisión de flujos comunicativos y culturales parece hoy abierto: si las TIC han provocado la convergencia de formatos y contenidos tanto informativos como de la industria cultural, las empresas de la comunicación en su mayoría pertenecientes a los países occidentales, han reaccionado con megaconglomerados en los que integran líneas de negocio tanto relacionadas con los mass media tradicionales como con el acceso y uso de la red.

\section{REFERENCIAS}

Adorno, T. W. y Horkheimer, M. (1969). Dialéctica de la I/ustración. Madrid: Trotta. 
Vaquerizo Domínguez, E. Medios de comunicación y flujos culturales internacionales: la vigencia actual del informe McBride

Amegaza, J. (2004). Televisión por satélite en el mundo: nuevos retos para las viejas lenguas. Barcelona: Portal de Comunicación de Universitat Autónoma de Barcelona. Recuperado de http://www.portalcomunicacion.com/dialeg/paper/pdf/16 amezaga.pdf

Castells, M. (1996). La era de la información. Vol. 1. La sociedad red. Madrid: Alianza Editorial. Recuperado de http://www.felsemiotica.org/site/wpcontent/uploads/2014/10/LA SOCIEDAD RED-Castells-copia.pdf

Castells, M. (1999). La era de la información. Vol. 2. El poder de la identidad. Madrid: Alianza Editorial.

CEPAL (Comisión Económica para América Latina y El Caribe). (2003). Los caminos hacia la sociedad de la información en América Latina y el Caribe. NIH Publication No LC/G.2195. Recuperado de https://www.cepal.org/publicaciones/xml/5/11575/DGE2195-CONF91-3.pdf

Dijk, V. J. (2006). Digital Divide Research. Achievements and Shortcomings. Poetics, 34(4), 221-235. Recuperado de https://pdfs.semanticscholar.org/db84/d71d147c18b68fb675df91baf0c02b4e4f91.pdf

Dijk, V. J. (2013). A Theory of the Digital Divide. The Digital Divide. In M. Ragnedda, \& G. W. Muschert (Eds.), The Digital Divide: The Internet and Social Inequality in International Perspective (pp. 29-51). New York: Routledge. Recuperado de https://research.utwente.nl/en/publications/a-theory-of-the-digital-divide

Esteinou, J. (2004). El Rescate del Informe Mc Bride y la Construcción de un Nuevo Orden Mundial de la Información. Razón y Palabra, 39, 1-15. Recuperado de http://www.razonypalabra.org.mx/anteriores/n39/jesteinou.html

García Canclini, N. (2017)._Maritza Urteaga y Néstor García Canclini conversan sobre la juventud en las ciencias sociales: delincuentes, consumidores, migrantes 0 actores alternativos. Revista Metamorfosis, Reina Sofía, 2-27. Recuperado de http://revistametamorfosis.es/index.php/metamorfosis/article/view/65

Giménez, G. (2009). Identidades sociales. Ciudad de México: Conaculta.

Gómez, R. (2016). Latino Television in the United States and Latin America: Addressing Networks, Dynamics, and Alliances. International. Journal of Communication, 10, 2811-2830. Recuperado de https://cutt.ly/rtfdbkm

Hall, S. (1972). The External Influences on Broadcasting. 'The External-Internal Dialectic in Broadcasting: Television's Double-Bind' Centre for Contemporary Cultural Studies. Discusion Paper. University of Birmingham. Recuperado de https://www.birmingham.ac.uk/Documents/college-artslaw/history/cccs/stencilledoccasional-papers/1to8and11to24and38to48/SOP04.pdf

Revista de Comunicación de la SEECI. 15 marzo, 2020 / 15 julio, 2020, nº 51, 43-62 
Vaquerizo Domínguez, E. Medios de comunicación y flujos culturales internacionales: la vigencia actual del informe McBride

Hall, S. y du Gay, P. (2003). Cuestiones de identidad cultural. Buenos Aires: Amorrortu. Recuperado de https://antroporecursos. files.wordpress.com/2009/03/hall-s-du-gay-p-1996cuestiones-de-identidad-cultural.pdf

Hopenhayn, M. (2004)._Orden Mediático y Orden Cultural: Una ecuación en busca de Resolución. Pensar Iberoamérica. Revista de cultura, 5. Recuperado de https://www.oei.es/historico/pensariberoamerica/ric05a02.htm

Jenkins, H. (2008). Convergence culture. La cultura de la convergencia de los medios de comunicación. Barcelona: Paidós.

Lévy, P. (2007). Cibercultura. La cultura de la sociedad digital. Barcelona: Antrophos. Recuperado de https://antroporecursos.files.wordpress.com/2009/03/levy-p-1997cibercultura.pdf

Marketing91 (2016). Top TV Channels in the world. Retrieved from https://www.marketing91.com/top-tv-channels-in-the-world/

Martín Barbero, J. (2002). Desencuentros de la sociabilidad y reencantamiento de la identidad. Analisi, 29, 45-62. Recuperado de https://www.raco.cat/index.php/Analisi/article/viewFile/15118/14959

McBride, S. (1980). Un solo mundo, voces múltiples. Ciudad de México: UnescoFondo de Cultura Económica.

Nielsen Report (2017). State of the Media- audio Today: Ethnic Audiences. Recuperado de http://www.nielsen.com/us/en/insights/reports/2017/state-of-themedia-audio-today-ethnic-audiences.html

Sandoval, E. A. (2007). Cibersocioantropología de comunidades virtuales. Revista Argentina de Sociología, 5(9). Recuperado de http://www.redalyc.org/pdf/269/26950905.pdf

Segovia Alonso, A. I. (2004). Una revisión histórica del proceso de concentración multimedia en Estados Unidos. Revista Comunicación y Sociedad, 2, 99-123. Recuperado de http://publicaciones.cucsh.udg.mx/pperiod/comsoc/pdf/2 2004/99-123.pdf

Segovia Alonso, A. I. (2005). La globalización y el mito del tamaño en la industria mediática y de telecomunicaciones. Anuario ININCO: Investigaciones de la Comunicación, 17(1), 243-266. Recuperado de https://www.academia.edu/12028759/La globalizaci\%C3\%B3n y el mito del ta ma\%C3\%B10 en la industria medi\%C3\%A1tica y de telecomunicaciones 
Vaquerizo Domínguez, E. Medios de comunicación y flujos culturales internacionales: la vigencia actual del informe McBride

Tello, E. (2007). Las tecnologías de la información y comunicaciones (TIC) y la brecha digital. Revista de Universidad y Sociedad de Conocimiento, 4(2), 1-8. Recuperado de https://www.raco.cat/index.php/Rusc/article/viewFile/78534/102611 https://journals.openedition.org/questionsdecommunication/1918

Thussu, D. K. (2007). Media on the Move: Global Flow and Contra-Flow. New York: Routledge. Retrieved from https://is.muni.cz/el/1423/podzim2016/ZUR393j/um/Media On the Move.pdf

Thompson, J. (1998). Los medios y la modernidad. Una teoría de los medios de comunicación. Barcelona: Ediciones Paidós.

Travesedo, C. (2014). Interculturalidad e información internacional. El gran desafío en el siglo mestizo. Historia y Comunicación Social, 19(3) 539-550. Recuperado de revistas.ucm.es/index.php/hics/article/download/45154/42516

Tulloch, C. D. (2009). La comunicación Sur-Sur: TeleSur, Al Yazeera y su impacto en el flujo de la información transnacional. IX Congreso IBERCOM, Sevilla-Cádiz. Recuperado de https://idus.us.es/xmlui/bitstream/handle/11441/34194/Flash124.pdf?sequence=1

Zenith Media Report (2017). Top 30 Global Media Owners 2017. Retrieved from https://www.zenithusa.com/top-30-global-media-owners-2017/

Zorogastua, J. (2015). Medios, publicidad e integración. La inmigración y los nuevos escenarios comunicativos en España 2000-2012. Tesis doctoral. Universidad CEU San Pablo. Madrid. Recuperado de http://dspace.ceu.es/bitstream/10637/8087/1/Medios JessicaZorogastua CEUTesi s 2016.pdf

\section{AUTOR:}

\section{Enrique Vaquerizo Domínguez}

Doctor en Comunicación Audiovisual por la Universidad Complutense de Madrid y Licenciado en Periodismo e Historia por la Universidad de Sevilla, ejerce como periodista y consultor de Comunicación Social y Corporativa además de como profesor del máster de Comunicación Política e Institucional de la Universidad Camilo José Cela. Ha publicado artículos en revistas nacionales e internacionales y ha participado en diversos libros colectivos, congresos y conferencias relacionados con las temáticas de migración, comunicación y TIC.

enrvaque@ucm.com

Orcid ID: https://orcid.org/0000-0002-4146-9900

Google Scholar: https://scholar.google.com/citations?user=yy9GQ6wAAAJ

Academia.edu:

https://independent.academia.edu/EnriqueVaquerizoDom\%C3\%ADnguez 